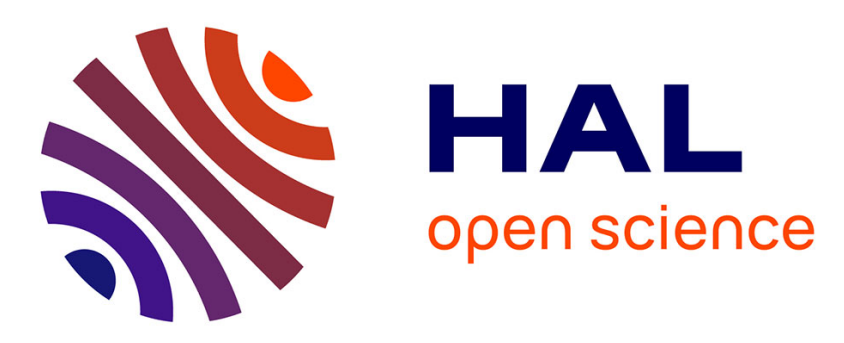

\title{
Stability crossing boundaries and fragility characterization of PID controllers for SISO systems with I/O Delays.
}

Irinel-Constantin Morarescu, César Fernando Méndez Barrios, Silviu-Iulian Niculescu, Keqin Gu

\section{To cite this version:}

Irinel-Constantin Morarescu, César Fernando Méndez Barrios, Silviu-Iulian Niculescu, Keqin Gu. Stability crossing boundaries and fragility characterization of PID controllers for SISO systems with I/O Delays.. 2011 American Control Conference (ACC), Jun 2011, San Francisco, United States. pp.4988 - 4993, 10.1109/acc.2011.5991530 . hal-00637998

\section{HAL Id: hal-00637998 \\ https://hal.science/hal-00637998}

Submitted on 3 Nov 2011

HAL is a multi-disciplinary open access archive for the deposit and dissemination of scientific research documents, whether they are published or not. The documents may come from teaching and research institutions in France or abroad, or from public or private research centers.
L'archive ouverte pluridisciplinaire HAL, est destinée au dépôt et à la diffusion de documents scientifiques de niveau recherche, publiés ou non, émanant des établissements d'enseignement et de recherche français ou étrangers, des laboratoires publics ou privés. 


\title{
Stability Crossing Boundaries and Fragility Characterization of PID Controllers for SISO Systems with I/O Delays
}

\author{
Irinel-Constantin Morărescu, César-Fernando Méndez-Barrios, Silviu-Iulian \\ Niculescu and Keqin $\mathrm{Gu}$
}

\begin{abstract}
This paper focuses on the closed-loop stability analysis of single-input-single-output (SISO) systems subject to input (or output) delays in the presence of PID-controllers. More precisely, using a geometric approach, we present a simple and user-friendly method for the closed-loop stability analysis as well as for the fragility of such PID controllers. The proposed approach is illustrated on several examples encountered in the control literature.
\end{abstract}

\section{Index Terms}

PID, Delay, SISO, Fragility, Geometry

\section{INTRODUCTION}

As reported in the literature [4], [23], [26], more than 95\% of the control-loops in the paper industries are controlled by SISO PID controllers. The "popularity" of PID controllers [3], [28]

I.-C. Morărescu is with Nancy-Université, CRAN UMR-CNRS 7039, 54516 Vandoeuvre Cedex, France.constantin.morarescu@ensem.inpl-nancy. fr

S.-I. Niculescu is with L2S (UMR CNRS 8506), CNRS-Supélec, 3, rue Joliot Curie, 91192, Gif-sur-Yvette, France. Silviu.Niculesculls. supelec.fr

$\mathrm{K}$. $\mathrm{Gu}$ is with the Department of Mechanical and Industrial Engineering, Southern Illinois University at Edwarsville, Edwarsville, IL 62026, USA. kgu@ siue.edu

C.F. Méndez-Barrios is with L2S (UMR CNRS 8506), CNRS-Supélec, 3, rue Joliot Curie, 91192, Gif-sur-Yvette, France. mendez@lss.supelec.fr. His work is financially supported by CONACyT, Mexico. 
can be attributed to their particular distinct features: simplicity and easy implementation. A long list of PID tuning methods for controlling industrial processes can be found in the literature, see, e.g., [23], [3], [26], to cite only a few. For further discussions in the case of systems with I/O delays, we refer to [26], [30], and the references therein.

This paper focuses on the design of PID controllers for SISO systems in the presence of I/O delays. The problem received a lot of attention in delay free systems, see, e.g., [14] (robustness techniques design leading to fragile controllers), [9] (non-fragile PID control design procedure), [2] (appropriate index to measure the fragility of PID controllers). In this context of delay free systems, some remarks concerning the controller robustness via coprime factorization and robustness optimization tools can be found in [15], [13]. However, there exists only a few results in the delay case as, for example, [27], where only (stable) first-order systems were considered, the authors in [16] proposed a non-fragile controller design for a linearized TCP/AQM model, more recently, based on the D-composition method, the properties regarding the increase in the number of unstable poles across the boundary of the PID gain were studied in [25], whereas in [12], the lines that contain the boundary of the stabilizing gain set for the ID (integral-derivative) plane are obtained, finally, based on the extension of the Hermite-Biehler theorem, in [24] a method to compute the set of stabilizing PID gain is obtained, but it requires much computation.

In this paper, inspired by the geometric ideas developed by Gu et al. [8] we start by developing a simple method to derive the stability regions in the gain parameters space of a PID-controller for a SISO system subject to (constant) time-delay. And next, we propose a simple algorithm to analyze the fragility of a given PID-controller for any SISO system subject to I/O delays. The method is based on the Implicit Function Theorem [10] and related properties, and requires three "ingredients":

(i) the construction of the stability crossing boundaries (surfaces) in the parameter-space defined by "P" (proportional), "I" (integral) and "D" (derivative) gains,

(ii) the explicit computation of the crossing direction (towards stability or instability) when such a surface is traversed,

(iii) finally, the explicit computation of the distance of some point to the closest stability crossing boundaries.

In the procedure above, the first step sends back to the $D$-decomposition method suggested 
by Neimark [22] in the 40s (see [18] for further comments) or to the parameter space approach (see, for instance, [1], [6] or [26] and the references therein). In the sequel, the stability crossing boundaries (surfaces for PID, curves for PI or PD controllers) represent the collection of all points for which the corresponding characteristic equation of the closed-loop system has at least one root on the imaginary axis. These boundaries define a "partition" of the parameter-space in several regions, each region having a constant number of unstable roots for all the parameters inside the region. Next, using an argument based on implicit function theorem one derives if a region has more or fewer unstable roots compared with its neighboring regions. This allows to detect the regions with no unstable roots which correspond to controller gains guaranteing the stability of the closed-loop system. This methodology has also advantages from the robustness point of view. Precisely, choosing controller gains inside a stability region and far from all the stability crossing boundaries that bound the region, the stability of the closed-loop system is ensured even for some small bounded variations of the controller gains.

The remaining part of the paper is organized as follows: the problem formulation and the class of systems under consideration are presented in Section II. The procedure to derive the stability crossing boundaries is described in Section III. In Section III-A we derive the frequencies for which a crossing surface may be traversed and in Section III-B we classify the boundaries obtained in III. Section III-C present the methodology which enables us to derive if crossing a stability surface in a given direction the closed-loop system gains or looses some unstable roots. In Section IV, the algorithm to analyze the fragility of a given PID controller is presented. Section V presents some illustrative examples and concluding remarks end the paper.

\section{THE FREQUENCY MODEL}

For the sake of brevity, let us consider now the class of strictly proper SISO open-loop systems with I/O delays given by the transfer function:

$$
G(s):=\frac{P(s)}{Q(s)} e^{-s \tau}=c^{T}\left(s I_{n}-A\right)^{-1} b e^{-s \tau},
$$

where $\left(A, b, c^{T}\right)$ is a state-space representation of the open-loop system. As mentioned in the Introduction, our aim is two-fold. First, design a PID controller

$$
C(s)=k\left(1+T_{d} s+\frac{1}{T_{i} s}\right)=k_{p}+k_{d} s+\frac{k_{i}}{s}
$$


that stabilizes the plant (1). Our second goal, is to derive an appropriate PID controller $\left(k_{p}^{*}, k_{d}^{*}, k_{i}^{*}\right)$ and the largest positive value $d$ such that the controller (2) stabilizes the system (1) for any $k_{p}$, $k_{d}$ and $k_{i}$, as long as

$$
\sqrt{\left(k_{p}-k_{p}^{*}\right)^{2}+\left(k_{d}-k_{d}^{*}\right)^{2}+\left(k_{i}-k_{i}^{*}\right)^{2}}<d .
$$

It is clear that the closed-loop dynamics is characterized by the equation:

$$
1+G(s) C(s)=0
$$

which rewrites as:

$$
f\left(s ; k_{p}, k_{d}, k_{i}\right):=\frac{1}{G(s)}+\left(k_{p}+k_{d} s+\frac{k_{i}}{s}\right)=0 .
$$

Our approach follows the lines presented in [17], [20]-[21]. More precisely, we want to derive the stability crossing boundaries $\mathcal{T}$ which is the set of parameters $\left(k_{p}, k_{d}, k_{i}\right) \in \mathbb{R}_{+}^{3}$ such that (4) has imaginary solutions. As the parameters $\left(k_{p}, k_{d}, k_{i}\right)$ cross the stability crossing boundaries, some characteristic roots cross the imaginary axis. We also consider $\Omega=\left\{\omega \in \mathbb{R} \mid \exists\left(k_{p}, k_{d}, k_{i}\right) \in\right.$ $\mathbb{R}_{+}^{3}$ such that $\left.f\left(j \omega ; k_{p}, k_{d}, k_{i}\right)=0\right\}$ the set of frequencies where the number of unstable roots of (4) changes. The set $\Omega$ will be called stability crossing set.

\section{StABILITY CROSSING CHARACTERIZATIONS}

Considering that $\Omega$ is known, the stability crossing boundaries are simply characterized by:

Proposition 1: The stability crossing boundaries associated to (4) are described as follows:

$$
\left\{\begin{array}{l}
k_{p}=-\Re\left(\frac{Q(j \omega)}{P(j \omega)} e^{j \omega \tau}\right) \\
k_{i}=k_{d} \omega^{2}+\omega \Im\left(\frac{Q(j \omega)}{P(j \omega)} e^{j \omega \tau}\right)
\end{array}, \quad \forall \omega \in \Omega .\right.
$$

Remark 1: For any fixed $\omega^{*} \in \Omega$, one obtains a section of a stability crossing surface which consists in a straight line parallel to the $\left(k_{d}, k_{i}\right)$ plane and passing through the point $\left(-\Re\left(\frac{Q(j \omega)}{P(j \omega)} e^{j \omega \tau}\right), 0, \omega \Im\left(\frac{Q(j \omega)}{P(j \omega)} e^{j \omega \tau}\right)\right)$. The slope of this line in the $\left(k_{d}, k_{i}\right)$ plane is always positive and is given by $\omega^{2}$.

Remark 2: From the Proposition 1 it is clear that $k_{i}=0$ represents a boundary.

Remark 3: Let the relative degree of the system (1) be $\rho=1$. Then, the closed-loop system (1) becomes a system of neutral-type (see, e.g., [11], [18]) and

$$
\left(k_{p},\left|\frac{q_{n}}{p_{n-1}}\right|, k i\right) \text { and }\left(k_{p},-\left|\frac{q_{n}}{p_{n-1}}\right|, k i\right)
$$


belong to the stability crossing surfaces. Here, $p_{n-1}$ and $q_{n}$ represent the leading coefficients of the polynomials $P(s)$ and $Q(s)$, respectively.

\section{A. Stability crossing sets}

In the sequel, we present a practical methodology to derive the stability crossing set. For the sake of brevity, we suppose the following technical assumption is satisfied:

Assumption 1: There exist some bounds $\left(\underline{k_{p}^{*}}, \overline{k_{p}^{*}}\right),\left(\underline{k_{d}^{*}}, \overline{k_{d}^{*}}\right)$ and $\left(\underline{k_{i}^{*}}, \overline{k_{i}^{*}}\right)$ of the controller gains.

These bounds can be arbitrarily fixed and, in principle, they are chosen by the designer according to the physical constraints of the model/controller. In this context, when Assumption 1 holds, the section of the stability crossing surface obtained for a fixed $\omega \in \Omega$ reduces to a segment (see Remark 1).

Proposition 2: Consider that Assumption 1 holds. Then the stability crossing set $\Omega$ is a union of bounded intervals consisting in all frequencies that simultaneously satisfy the following conditions:

$$
\left\{\begin{array}{l}
\underline{k_{p}^{*}} \leq-\Re\left(\frac{Q(j \omega)}{P(j \omega)} e^{j \omega \tau}\right) \leq \overline{k_{p}^{*}} \\
\exists \underline{k_{d}^{*}} \leq k_{d} \leq \overline{k_{d}^{*}} \text { s.t. } \underline{k_{i}^{*}} \leq k_{d} \omega^{2}+\omega \Im\left(\frac{Q(j \omega)}{P(j \omega)} e^{j \omega \tau}\right) \leq \overline{k_{i}^{*}} .
\end{array}\right.
$$

Remark 4: Propositions 1 and 2 lead to the following algorithm to determine both the stability crossing set $\Omega$ and the stability crossing boundaries $\mathcal{T}$ :

- Step 1: One solves the system $\underline{k_{p}^{*}} \leq-\Re \frac{1}{G(j \omega)} \leq \overline{k_{p}^{*}}$ getting a union of intervals.

- Step 2: For all $\omega$ derived at the previous step one computes $k_{p}$ and derive the equation of the line $\left(k_{d}, k_{i}\right)$ given by the second equation in (5).

- Step 3: Finally, one keeps only those frequencies $\omega$ for which the line $\left(k_{d}, k_{i}\right)$ derived at the previous step intersects the rectangle $\left[\left(\underline{k_{d}^{*}}, \underline{k_{i}^{*}}\right) ;\left(\overline{k_{d}^{*}}, \underline{k_{i}^{*}}\right) ;\left(\overline{k_{d}^{*}}, \overline{k_{i}^{*}}\right) ;\left(\underline{k_{d}^{*}}, \overline{k_{i}^{*}}\right)\right]$.

Consider now, that either $k_{d}$ or $k_{i}$ is fixed. Let us also denote by $\mathcal{T}_{h}, h \in\{i, d\}$ the crossing curve when $d$ or $i$ is fixed and consider the following decomposition into real and imaginary 
parts:

$$
\begin{aligned}
& R_{0}+j I_{0}=\left.j \frac{\partial f\left(s ; k_{p}, k_{h}\right)}{\partial s}\right|_{s=j \omega}, \\
& R_{1}+j I_{1}=-\left.\frac{\partial f\left(s ; k_{p}, k_{h}\right)}{\partial k_{h}}\right|_{s=j \omega}, \\
& R_{2}+j I_{2}=-\left.\frac{\partial f\left(s ; k_{p}, k_{h}\right)}{\partial k_{p}}\right|_{s=j \omega} .
\end{aligned}
$$

Then, since $f\left(s ; k_{p}, k_{h}\right)$ is an analytic function of $s, k_{p}$ and $k_{h}$, the implicit function theorem indicates that the tangent of $\mathcal{T}_{h}$ can be expressed as

$$
\begin{aligned}
\left(\begin{array}{c}
\frac{d k_{p}}{d \omega} \\
\frac{d k_{h}}{d \omega}
\end{array}\right) & =\left(\begin{array}{cc}
R_{2} & R_{1} \\
I_{2} & I_{1}
\end{array}\right)^{-1}\left(\begin{array}{c}
R_{0} \\
I_{0}
\end{array}\right) \\
& =\frac{1}{R_{1} I_{2}-R_{2} I_{1}}\left(\begin{array}{c}
R_{1} I_{0}-R_{0} I_{1} \\
R_{0} I_{2}-R_{2} I_{0}
\end{array}\right),
\end{aligned}
$$

provided that

$$
R_{1} I_{2}-R_{2} I_{1} \neq 0
$$

It follows that $\mathcal{T}_{h}$ is smooth everywhere except possibly at the points where either (11) is not satisfied, or when

$$
\frac{d k_{p}}{d \omega}=\frac{d k_{h}}{d \omega}=0
$$

Remark 5: If (12) is satisfied, then straightforward computations show us that $R_{0}=I_{0}=0$. In other words, $s=j \omega$ is a multiple solution of (15).

\section{B. Classification of the stability crossing boundaries}

It is worth noting here that $k_{p}, k_{d}$ and $k_{i}$ continuously depend on $\omega$. Therefore, in order to classify the stability crossing boundaries we will first classify the intervals belonging to the stability crossing set. Precisely, a deeper analysis of Proposition 2 allows us to say that $\omega^{*}$ is an end of an interval belonging to $\Omega$ if and only if one of the following condition is satisfied:

- Type 1: $-\Re \frac{1}{G\left(j \omega^{*}\right)}=k_{p}^{*}$, where $k_{p}^{*}$ is either $k_{p}^{*}=\underline{k_{p}^{*}}$ or $k_{p}^{*}=\overline{k_{p}^{*}}$. In this case, $\omega^{*} \in \Omega$ and the stability crossing surface approach a segment parallel to the $\left(k_{d}, k_{i}\right)$ plane given by 
$k_{p}=k_{p}^{*}$ and

$$
\begin{gathered}
k_{i}=k_{d} \cdot\left(\omega^{*}\right)^{2}+\omega^{*} \Im \frac{1}{G\left(j \omega^{*}\right)}, \\
\underline{k_{d}^{*}} \leq k_{d} \leq \overline{k_{d}^{*}}, \quad \underline{k_{i}^{*}} \leq k_{i} \leq \overline{k_{i}^{*}}
\end{gathered}
$$

- Type 2: $-\frac{1}{\omega^{*}} \Im \frac{1}{G\left(j \omega^{*}\right)}=k_{d}^{*}$. In this case $\omega^{*} \in \Omega$ and the stability crossing surface ends in the point $\left(-\Re \frac{1}{G\left(j \omega^{*}\right)},-\frac{1}{\omega^{*}} \Im \frac{1}{G\left(j \omega^{*}\right)}, 0\right)$, included in the $\left(k_{p}, k_{d}\right)$ plane.

- Type 3: $\omega^{*} \Im \frac{1}{G\left(j \omega^{*}\right)}=k_{i}^{*}$. In this case $\omega^{*} \in \Omega$ and the stability crossing surface ends in the point $\left(-\Re \frac{1}{G\left(j \omega^{*}\right)}, 0, \omega^{*} \Im \frac{1}{G\left(j \omega^{*}\right)}\right)$, included in the $\left(k_{p}, k_{i}\right)$ plane.

Similarly to [8], we classify the stability crossing boundaries in 8 types in function of the kind of the left and right ends of the corresponding frequency crossing interval. Precisely, we say that a crossing surface is of type $a b, a, b \in\{1,2,3\}$ if it corresponds to a crossing interval $\left(\omega_{l}, \omega_{r}\right)$ with $\omega_{l}$ of type $a$ and $\omega_{r}$ of type $b$. Let us notice that generally the intervals $\left(\omega_{l}, \omega_{r}\right)$ are closed.

\section{Crossing direction}

As explained in [7], [29], a pair of imaginary zeros $(\bar{s}, s)$ of the characteristic equation $f\left(s ; k_{p}, k_{d}, k_{i}\right)=0$ cross the imaginary axis through the gates $-j \omega, j \omega$ respectively, as $\left(k_{p}, k_{d}, k_{i}\right)$ moves from one side of a stability crossing surface to the other side. The direction of crossing may be calculated using implicit function theorem as described in [8], [19]. Precisely, the characteristic equation $f\left(s ; k_{p}, k_{d}, k_{i}\right)=0$ defines an implicit function $s$ of variables $k_{p}, k_{d}$ and $k_{i}$. The definition of $f\left(s ; k_{p}, k_{d}, k_{i}\right)$ given by (4) allows us to compute the following partial derivatives:

$$
\begin{aligned}
\frac{\partial s}{\partial k_{p}} & =\frac{s^{2} G^{2}(s)}{k_{i} G^{2}(s)-k_{d} s^{2} G^{2}(s)+s^{2} G^{\prime}(s)}, \\
\frac{\partial s}{\partial k_{d}} & =\frac{s^{3} G^{2}(s)}{k_{i} G^{2}(s)-k_{d} s^{2} G^{2}(s)+s^{2} G^{\prime}(s)}, \\
\frac{\partial s}{\partial k_{i}} & =\frac{s G^{2}(s)}{k_{i} G^{2}(s)-k_{d} s^{2} G^{2}(s)+s^{2} G^{\prime}(s)} .
\end{aligned}
$$

Let $\left(\bar{k}_{p}, \bar{k}_{d}, \bar{k}_{i}\right)$ a point belonging to a stability crossing surface and let $s=j \bar{\omega}, \bar{\omega}>0$ be the corresponding imaginary zero of the characteristic equation. Let $\mathbf{x}=\left(x_{p}, x_{d}, x_{i}\right)$ be a unit vector 
that is not tangent to the surface. Let us also use the following notation $\vec{k}=\left(k_{p}, k_{d}, k_{i}\right)$ and $\vec{k}_{*}=\left(\bar{k}_{p}, \bar{k}_{d}, \bar{k}_{i}\right)$.

Proposition 3: A pair of zeros of (4) moves from the left half complex plane (LHP) to the right half complex plane (RHP) as $\left(k_{p}, k_{d}, k_{i}\right)$ moves from one side of a stability crossing surface to the other side through $\left(\bar{k}_{p}, \bar{k}_{d}, \bar{k}_{i}\right)$ in the direction of $\mathbf{x}$ if

$$
\left.\Re\left(\frac{\partial s}{\partial k_{p}} x_{p}+\frac{\partial s}{\partial k_{d}} x_{d}+\frac{\partial s}{\partial k_{i}} x_{i}\right)\right|_{s=j \bar{\omega}, \vec{k}=\vec{k}_{*}}>0 .
$$

The crossing is from the RHP to the LHP if the inequality (14) is reversed.

\section{Fragility Analysis of PID CONTROllers}

Consider now the PID fragility problem, that is the problem of computing the maximum controller parameters deviation without loosing the closed-loop stability. In other words, given the parameters $\left(k_{p}^{*}, k_{d}^{*}, k_{i}^{*}\right)$ such that the roots of the closed-loop characteristic equation:

$$
Q(s)+P(s)\left(k_{p}^{*}+k_{d}^{*} s+\frac{k_{i}^{*}}{s}\right) e^{-s \tau}=0,
$$

are located in $\mathbb{C}_{-}$(that is the closed-loop system is asymptotically stable), find the maximum parameter deviation $d \in \mathbb{R}_{+}$such that the roots of (3) stay located in $\mathbb{C}_{-}$for all controllers $\left(k_{p}, k_{d}, k_{i}\right)$ satisfying:

$$
\sqrt{\left(k_{p}-k_{p}^{*}\right)^{2}+\left(k_{d}-k_{d}^{*}\right)^{2}+\left(k_{i}-k_{i}^{*}\right)^{2}}<d .
$$

This problem can be more generally reformulated as: find the maximum parameter deviation $d$ such that the number of unstable roots of (3) remains unchanged.

First, let us introduce some notation:

$$
\begin{aligned}
\mathcal{T} & =\bigcup_{l=1}^{N} \mathcal{T}_{l}, \quad \mathcal{T}_{l}=\left\{\left(k_{p}, k_{d}, k_{i}\right) \mid \omega \in \Omega_{l}\right\}, \\
\overrightarrow{k(\omega)} & =\left(k_{p}(\omega), k_{d}(\omega), k_{i}(\omega)\right)^{T}, \quad \overrightarrow{k^{*}}=\left(k_{p}^{*}, k_{d}^{*}, k_{i}^{*}\right)^{T}, \\
\overrightarrow{k_{a b}}(\omega) & =\left(k_{a}(\omega), k_{b}(\omega)\right)^{T}, \quad \overrightarrow{k_{a b}^{*}}=\left(k_{a}^{*}, k_{b}^{*}\right)^{T},
\end{aligned}
$$

where $a, b \in\{p, i, d\}$. Let us also denote $d_{\mathcal{T}}=\min _{l \in\{1, \ldots, N\}} d_{l}$, where

$$
d_{l}=\min _{\left(k_{p}, k_{d}, k_{i}\right) \in \mathcal{T}_{l}}\left\{\sqrt{\left(k_{p}-k_{p}^{*}\right)^{2}+\left(k_{d}-k_{d}^{*}\right)^{2}+\left(k_{i}-k_{i}^{*}\right)^{2}}\right\} .
$$




\section{A. PI-PD Controller Fragility}

Let $k_{d}=k_{d}^{*} \in \mathbb{R}$ or $k_{i}=k_{i}^{*} \in \mathbb{R}$ be fixed, we have the following result:

Proposition 4: The maximum parameter deviation, without changing the number of unstable roots of the closed-loop equation (3) can be expressed as:

PI-Controller: Let $k_{d}=k_{d}^{*}$ be fixed,

$$
d_{p i}^{*}=\min \left\{\left|k_{i}^{*}\right|, \min _{\omega \in \Omega_{f_{p i}}}\left\{\left\|\overrightarrow{k_{p i}}(\omega)-\overrightarrow{k_{p i}^{*}}\right\|\right\}\right\} .
$$

PD-Controller: Let $k_{i}=k_{i}^{*}$ be fixed, then $d_{p d}^{*}=$

$$
d_{p d}^{*}=\min \left\{k_{d \infty},\left|k_{p}^{*}-k_{p}(0)\right|, \min _{\omega \in \Omega_{f_{p d}}}\left\{\| \overrightarrow{k_{p d}}(\omega)-\overrightarrow{k_{p d}^{*}}||\right\}\right\},
$$

with,

$$
k_{d \infty}= \begin{cases}\min \left\{\left|k_{d}^{*}-\right| \frac{q_{n}}{p_{m}}||,\left|k_{d}^{*}+\right| \frac{q_{n}}{p_{m}}||\right\} & \text { if } \quad m=n-1 \\ \emptyset & \text { if } \quad m<n-1\end{cases}
$$

and $\Omega_{f_{a b}}, a, b \in\{p, i, d\}$ is the set of roots of the function $f_{a b}: \mathbb{R}_{+} \mapsto \mathbb{R}$,

$$
f_{a b}(\omega) \triangleq\left\langle\left(\overrightarrow{k_{a b}}(\omega)-\overrightarrow{k_{a b}^{*}}\right), \frac{d \overrightarrow{k_{a b}}(\omega)}{d \omega}\right\rangle,
$$

where " $\langle\cdot, \cdot\rangle "$ means the inner product.

\section{B. DI Projection}

Let $k_{p}=k_{p}^{*} \in \mathbb{R}$ be fixed, we have the following result:

Proposition 5: The maximum parameter deviation from $\left(k_{d}^{*}, k_{i}^{*}\right)$, without changing the number of unstable roots of the closed-loop equation (3) can be expressed as:

$$
d_{d i}^{*}=\min \left\{\left|k_{i}^{*}\right|, \min _{\omega_{\ell} \in \Omega_{k_{p}^{*}}}\left\{\left|\frac{\omega_{\ell}^{2} k_{d}^{*}-k_{i}^{*}+\omega_{\ell} \Im\left\{\frac{Q\left(j \omega_{\ell}\right)}{P\left(j \omega_{\ell}\right)} e^{j \omega_{\ell} \tau}\right\}}{\sqrt{\left(\omega_{\ell}\right)^{4}+1}}\right|\right\},\right.
$$

where $\Omega_{k_{p}^{*}}$ is the set of roots of the function $f_{k_{p}^{*}}: \mathbb{R} \times \mathbb{R}_{+} \mapsto \mathbb{R}$,

$$
f_{k_{p}^{*}}\left(k_{p}^{*}, \omega\right) \triangleq k_{p}^{*}+\Re\left\{\frac{Q(j \omega)}{P(j \omega)} e^{j \omega \tau}\right\} .
$$

Remark 6: Observe that (20) has an uncountable number of solutions, however in Proposition 5 we have considered the set including the corresponding $\left(k_{d}^{*}, k_{i}^{*}\right)$ points. 


\section{PID Fragility Algorithm}

In order to obtain the obtain the PID fragility we present the following algorithm:

- Step 1: Let $k_{p i d}^{*} \in \mathbb{R}^{3}$ be fixed. Then, set $d=\min \left\{d_{p i}^{*}, d_{p d}^{*}, d_{d i}^{*}\right\}$.

- Step 2: Sweep over all $\theta \in\left[-\frac{\pi}{2}, \frac{\pi}{2}\right]$ and compute $k_{p \theta}^{*}=k_{p}^{*}+d \sin \theta$.

- Step 3: Solve $f_{k_{p}^{*}}\left(k_{p \theta}^{*}, \omega\right)=0$ and denote by $\Omega_{\theta}$ the set of solutions.

- Step 4: Compute,

$$
d_{\theta}^{*}=\min _{\omega_{\ell} \in \Omega_{\theta}}\left\{\left|\frac{\left(\omega_{\ell}\right)^{2} k_{d}^{*}-k_{i}^{*}+\omega_{\ell} \Im\left\{\frac{Q\left(j \omega_{\ell}\right)}{P\left(j \omega_{\ell}\right)} e^{j \omega_{\ell} \tau}\right\}}{\sqrt{\left(\omega_{\ell}\right)^{4}+1}}\right|\right\} .
$$

- Step 5: If $d_{\theta}^{*}<d \cos \theta$ then set $d=d_{\theta}^{*} / \cos \theta$ and go to step 2. Otherwise continue to step 2.

- Step 6: If $\theta=\frac{\pi}{2}$, the procedure is finish and $d$ is the PID fragility for the controller $\left(k_{p}^{*}, k_{d}^{*}, k_{i}^{*}\right)$.

\section{ILLUSTRATIVE EXAMPLES}

In order to motivate the previous results, we consider in the sequel some numerical examples.

\section{A. PID fragility analysis}

Example 1: Consider the following system [24]:

$$
G(s)=\frac{s^{3}-4 s^{2}+s+2}{s^{5}+8 s^{4}+32 s^{3}+46 s^{2}+46 s+17} e^{-s} .
$$

By choosing $k_{p}^{*} \in\left[0, \frac{9}{2}\right]$, we obtain the stability region depicted in Fig.1. Next, in order to illustrate the proposed PID fragility-algorithm, consider $\left(k_{p}^{*}, k_{d}^{*}, k_{i}^{*}\right)=(2,3,3)$, leading to the values in Table I and depicted in Fig.2.

TABLE I

PID FRAGILITY FOR THE EXAMPLE (21).

\begin{tabular}{|c|c|c|c|}
\hline $\begin{array}{c}\text { Controller } \\
\left(k_{p}^{*}, k_{d}^{*}, k_{i}^{*}\right)\end{array}$ & $\begin{array}{c}\text { Fragility } \\
(P I, P D, D I)\end{array}$ & $\begin{array}{c}\text { Initial } \\
\text { PID-Fragility }\end{array}$ & $\begin{array}{c}\text { PID-Fragility } \\
\min \left\{d^{*}, d_{\theta}^{*}\right\}\end{array}$ \\
\hline \hline & $d_{p i}^{*}=1.68051$ & & \\
$(2,3,3)$ & $d_{p d}^{*}=1.33313$ & $d^{*}=1.27520$ & $d_{\theta}^{*}=1.26295$ \\
& $d_{d i}^{*}=1.27520$ & & \\
\hline
\end{tabular}




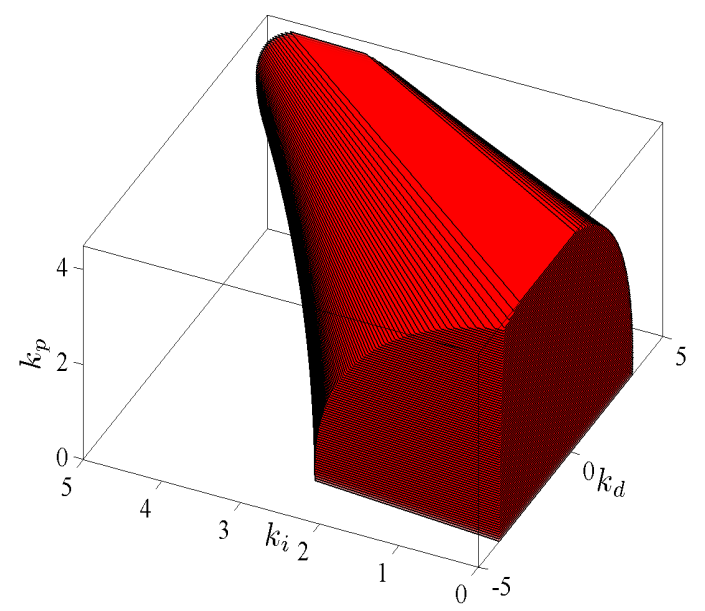

Fig. 1. The PID stability region for $k_{p} \in\left[0, \frac{9}{2}\right]$.

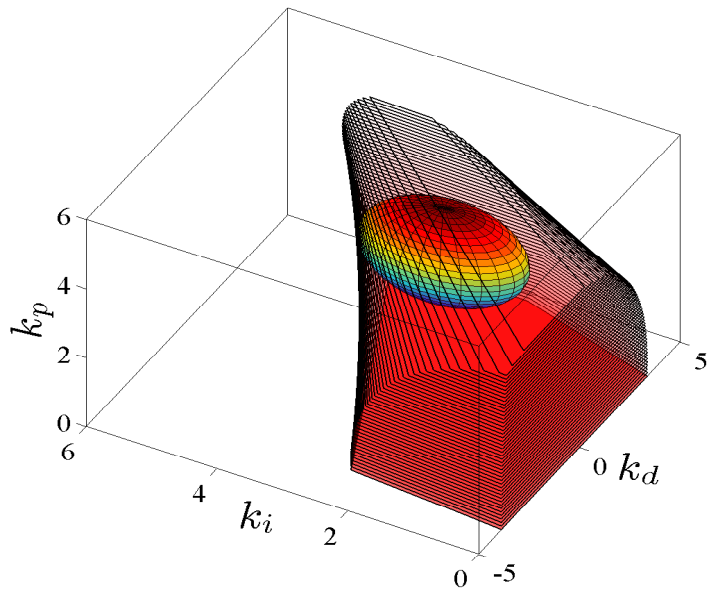

Fig. 2. PID-fragility for the controller $\left(k_{p}^{*}, k_{d}^{*}, k_{i}^{*}\right)=(2,3,3)$.

Example 2 (unstable, non-minimal phase system): Consider the following plant [17],

$$
G(s)=\frac{s-2}{s^{2}-\frac{1}{2} s+\frac{13}{4}} e^{-\frac{1}{2} s} .
$$

The interest in the analysis of this system, remains in the fact that the closed-loop plant becomes a system of Neutral-Type. Now, applying the same procedure as before, and considering $k_{p}^{*} \in$ $(0.32595,1.625)$ we obtain the following stability region.

For the fragility analysis, lets consider the controller $\left(k_{p}^{*}, k_{d}^{*}, k_{i}^{*}\right)=\left(\frac{5}{8},-\frac{1}{10},-\frac{2}{5}\right)$, leading to the results summarized in Table II. Figure 4 illustrate such a results. 


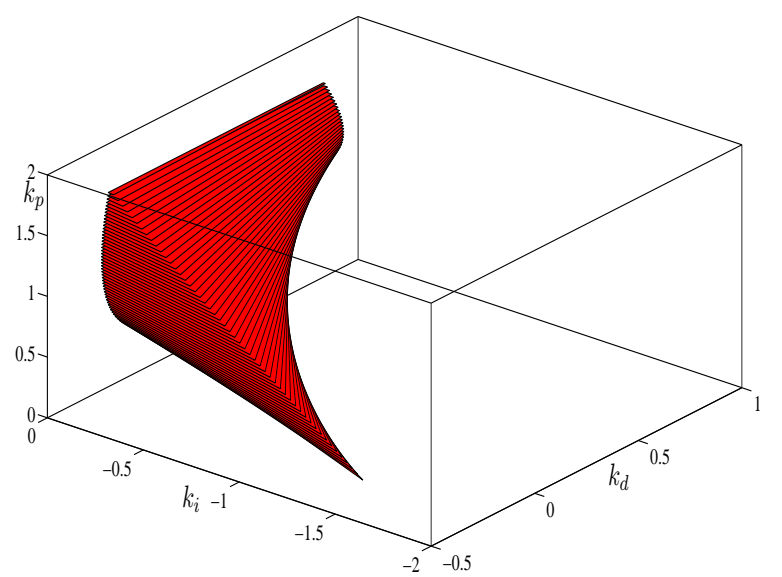

Fig. 3. The PID stability region of Neutral-Type .

TABLE II

PID FRAGILITY FOR THE EXAMPLE (22).

\begin{tabular}{|c|c|c|c|}
\hline $\begin{array}{c}\text { Controller } \\
\left(k_{p}^{*}, k_{d}^{*}, k_{i}^{*}\right)\end{array}$ & $\begin{array}{c}\text { Fragility } \\
(P I, P D, D I)\end{array}$ & $\begin{array}{c}\text { Initial } \\
\text { PID-Fragility }\end{array}$ & $\begin{array}{c}\text { PID-Fragility } \\
\min \left\{d^{*}, d_{\theta}^{*}\right\}\end{array}$ \\
\hline \hline & $d_{p i}^{*}=0.29314$ & & \\
$\left(\frac{5}{8}, \frac{-1}{10}, \frac{-2}{5}\right)$ & $d_{p d}^{*}=0.16758$ & $d^{*}=0.16758$ & $d_{\theta}^{*}=0.16453$ \\
& $d_{d i}^{*}=0.16782$ & & \\
\hline
\end{tabular}

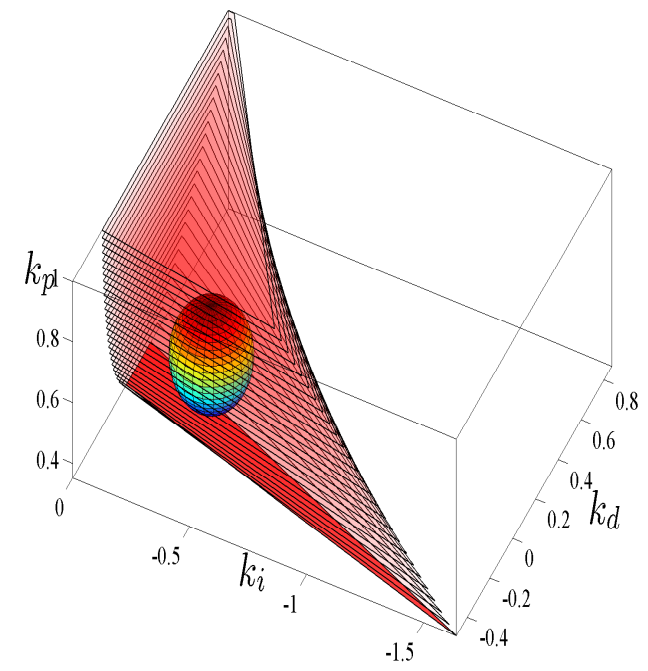

Fig. 4. PID-fragility for the controller $\left(k_{p}^{*}, k_{d}^{*}, k_{i}^{*}\right)=\left(\frac{5}{8},-\frac{1}{10},-\frac{2}{5}\right)$. 


\section{B. Stability crossing boundaries classification}

Example 3: Finally, lets consider the SISO plant [5],

$$
G(s)=\frac{-s^{4}-7 s^{3}-2 s+1}{(s+1)(s+2)(s+3)(s+4)\left(s^{2}+s+1\right)} e^{-\frac{1}{20} s} .
$$

By choosing the rectangle: $0 \leq k_{p} \leq 5,-12 \leq k_{i} \leq 5,0 \leq k_{d} \leq 10$, we obtain the following cases: Based in these results, the table III classifies the cases cited above.

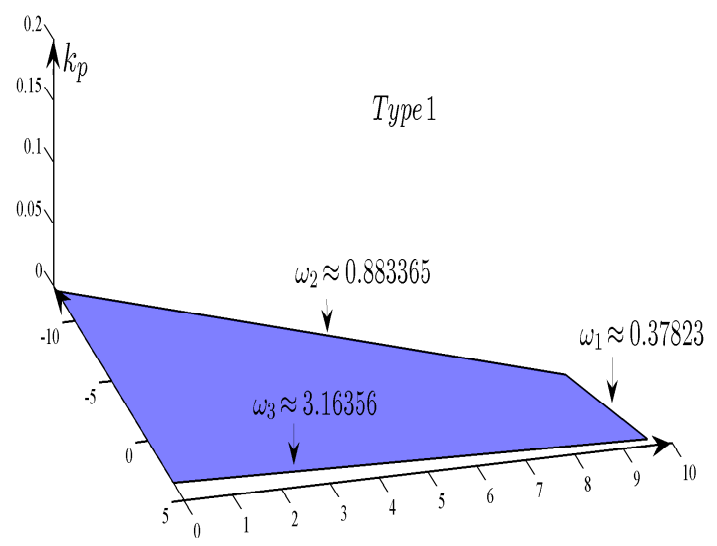

Fig. 5. Boundary classification Type 1 for the system (23).

TABLE III

CLASSIFICATION INTERVALS TYPE FOR THE SYSTEMS (23).

\begin{tabular}{cc}
\hline \hline Interval & Classification \\
\hline \hline$[0.37823,3.16356]$ & Type 11 \\
{$[0.37823,0.89290]$} & Type 12 \\
{$[0.37823,0.41294]$} & Type 13 \\
{$[0.89290,3.16356]$} & Type 21 \\
{$[0.41294,3.16356]$} & Type 31 \\
{$[0.41294,0.89290]$} & Type 32 \\
\hline \hline
\end{tabular}



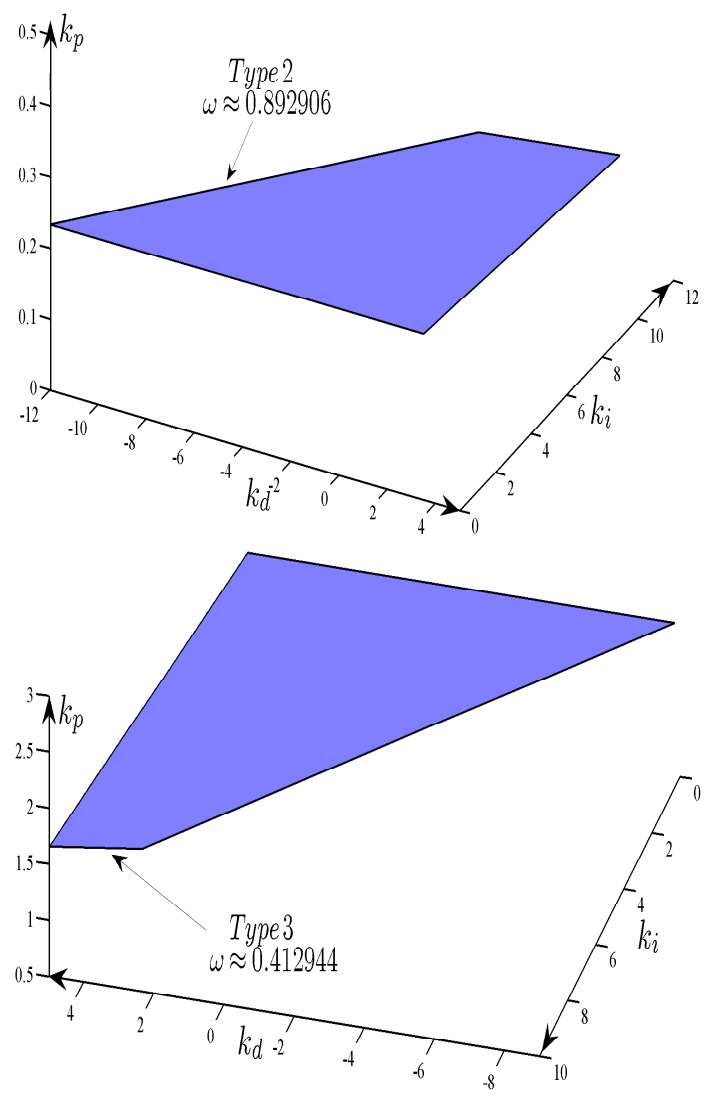

Fig. 6. Stability crossing boundaries classification for the system (23). (Upper) Type 2. (Lower) Type 3.

\section{CONCLUSIONS}

In this paper, we focused on stabilizing a class of SISO linear systems with constant delay in the input or output by using PID controllers. First, by exploiting the system properties we have characterized the stability crossing boundaries in the parameter-set defined by the controller's parameters. Second, we have developed a simple geometrical method to construct the PID stability region, that characterize the set of all stabilizing controller parameter. Finally, a simple geometric-based algorithm is derived for computing the fragility of PID-controllers. To prove the efficiency of the proposed methods, several illustrative examples have been considered. It is important to note that such an idea can be easily extended to proper SISO systems with I/O delays. 


\section{REFERENCES}

[1] Ackermann, J., Blee, P., Bünte, T., Güvenc, L., Kaesbauer, D., Kordt, M., Muhler, M. and Odenthal, D.: Robust control. The parameter space approach, Springer: London, 2002.

[2] Alfaro, V.M.: PID Controller's Fragility, ISA Transactions, vol.46, pp.555-559, 2007.

[3] Aström, K. J. and Hägglund, T.: PID Controllers: Theory, Design and Tunning, Instrument Society of America, 2nd edition, 1995.

[4] Aström, K. J. and Hägglund, T.: The future of PID control, Chem. Eng. Progress, vol.9, pp.1163-1175, 2001.

[5] Bajcinca, N.: Computation of stable regions in PID parameter space for time-delay systems, In W. Michiels(Ed.)., Proc. of 5th IFAC workshop on time-delay systems, Oxford:Elsevier, 2005.

[6] Bhattacharyya, S.P., Chapellat, H. and Keel, L.H.: Robust control. The parametric approach, Prentice Hall, 1995.

[7] Cooke, K. L. and van den Driessche, P.: On zeroes of some transcendental equations, Funkcialaj Ekvacioj, vol. 29, No.1, 7790, 1986.

[8] Gu, K., Niculescu, S.-I. and Chen, J.: On stability crossing curves for general systems with two delays in J. Math. Anal. Appl., vol. 311, pp. 231-253, 2005.

[9] Ho, M.T.: Non Fragile PID Controller Design, Proceeding of the 39th CDC, Sidney Australia, 2000.

[10] Guggenheimer, H.W.: Differential geometry (Dover: New York, 1977).

[11] Hale, J. K. and Verduyn Lunel, S. M.: Introduction to Functional Differential Equations (AMS, vol. 99, Springer-Verlag: New York, 1993).

[12] Hohenbichler, N.: All stabilizing PID controllers for time delay systems, Automatica, vol. 45, pp.2678-2684, 2009.

[13] Keel, L.H. and Bhattacharyya, S.P.: “Author's Reply,” IEEE Trans. Automat. Contr., vol. 43, pp.1268, 1998.

[14] Keel, L.H. and Bhattacharyya, S.P.: Robust, Fragile or Optimal, IEEE Trans. Automat. Contr., vol. 42, pp.1098-1105, 1997.

[15] Mäkilä, P.M.: Comments on: Robust, Fragile, or Optimal, IEEE Trans. Automat. Contr., vol. 43, pp.1265-1267, 1998.

[16] Melchor-Aguilar, D. and Niculescu, S.-I.: Robust non-fragile PI controllers for delay models of TCP/AQM networks, submitted, 2007

[17] Méndez-Barrios, C.-F., Niculescu, S.-I., Morărescu, I.-C. and Gu, K.: On the Fragility of PI Controllers for Time-Delay SISO systems - 16th Mediterranean Conference on Control and Automation, MED’08, 529-534, June 2008, Ajaccio, France.

[18] Michiels, W. and Niculescu, S.-I.: Stability and stabilization of time-delay systems. An eigenvalue-based approach, SIAM: Philadelphia, 2007.

[19] Morarescu, C.-I., Niculescu, S.-I. and Gu, K.: Stability crossing curves of shifted gamma-distributed delay systems, SIAM Journal on Applied Dynamical Systems, vol. 6, No 2, pp. 475-493, 2007.

[20] Morărescu, C.I. and Niculescu, S.-I.: Stability crossing curves of SISO systems controlled by delayed output feedback. Dynamics of Continuous, Discrete and Impulsive Systems, series B, vol. 14, No 5, pp. 659-678, 2007.

[21] Morărescu, I.-C., Niculescu, S.-I. and Gu, K.: On the geometry of PI controllers for SISO systems with input delays IFAC Workshop on Time Delay Systems, TDS'07, 17-19 Septembre 2007, Nantes, France.

[22] Neimark, J.: D-subdivisions and spaces of quasi-polynomials, Prikl. Math. Mech., vol. 13, pp.349-380, 1949.

[23] O’Dwyer, A.: PI and PID controller tuning rules for time delay process: a summary (Technical report AOD-00-01, Dublin Institute of Technology, Ireland, 2000).

[24] Ou, L.L., Zhang, W.D and Yu, L.: Low-Order Stabilization of LTI Systems With Time Delay, IEEE Trans. Automat. Contr., vol. 54(4), pp.774-787, 2010. 
[25] Saeki, M.: Properties of Stabilizing PID Gain Set in Parameter Space, IEEE Trans. Automat. Contr., vol. 52(9), pp.17101715, 2007.

[26] Silva, G.J., Datta, A., and Bhattacharrya, S.P.: PID Controllers for Time Delay Systems, Birkhäuser, Boston, 2005.

[27] Silva, G.J., Datta, A., and Bhattacharrya, S.P.: PID tuning revisited: Guaranteed stability and non-fragility, in Proc. American Contr. Conf., Anchorage, AK, 5000-5006, 2002.

[28] Tan, K.K., Wang, Q.G. and Hang, C.C.: Advances in PID control, Springer: Berlin, 1999.

[29] Walton, K. and Marshall, J. E.: Direct method for TDS stability analysis, IEEE Proc. 134, part D,101-107, 1987.

[30] Zhong, Q.-C.: Robust control of time-delay systems Springer: London, 2006. 